\title{
HLA-DP4 Antigen
}

National Cancer Institute

\section{Source}

National Cancer Institute. HLA-DP4 Antigen. NCI Thesaurus. Code C128310.

HLA-DP4 antigen is a protein complex of the major histocompatibility complex, class II DP protein in which the alpha subunit is encoded by the HLA-DPA1*0103 allele and the beta subunit serotype is encoded by either the *0401 allele or the *0402 allele of the HLA-DPB1 gene. This antigen represents the most common haplotype. 